\title{
Using a cartographic model to assist medical diagnoses
}

\author{
Elizabeth S. Carvalho • Adérito F. Marcos • \\ Maribel Y. Santos • João Espregueira-Mendes • \\ Rogério B. Pereira
}

Received: 10 January 2008 / Accepted: 27 March 2008

C CARS 2008

\begin{abstract}
Objective The main goal of the CHUB (cartographic human body) model is to introduce a cartographic approach that can assist the analysis, visualization and diagnosis of medical images and related data.

Method A cartographic model is proposed as a reference framework for the development of computer systems and applications. This model accommodates subtle spatial features of these data that may facilitate diagnosis. Medical images and related data are structured into different "human-referenced" information layers. Algebraic map operations are used to combine these layers and achieve greater insights into the information content.

Results Two case studies were considered to evaluate and validate the model: hydrokinetic therapy and the diagnosis of knee osteoarthritis. A prototype based on the CHUB model was implemented and two different approaches were performed to test and evaluate it. Full acceptance of the model was achieved by clinicians who used the CHUB system.
\end{abstract}

E. S. Carvalho ( $\varangle)$ · A. F. Marcos · M. Y. Santos

DSI, Departamento de Sistemas de Informação,

University of Minho, Escola de Engenharia, Guimarães, Portugal

e-mail: ecarvalho@dsi.uminho.pt

A. F. Marcos

e-mail: marcos@dsi.uminho.pt

M. Y. Santos

e-mail: maribel@dsi.uminho.pt

J. Espregueira-Mendes

Escola de Ciências da Saúde, University of Minho, Braga, Portugal

e-mail: espregueiramendes@ecsaude.uminho.pt

R. B. Pereira

Faculdade de Ciências da Saúde, University Fernando Pessoa,

Porto, Portugal

e-mail: Rogerio@ufp.pt
Conclusion A cartographic model provides a feasible means for analyzing and visualizing medical image and related data. CHUB is a suitable model that may be used as a common framework for systems, applications and/or support tools that analyze and visualize medical images and related data (for example, in disease diagnosis).

Keywords Visualization - Cartographic model . Hydrokinetic therapy $\cdot$ Osteoarthritis

\section{Introduction}

Nowadays, scientific information analysis [5] and visualization offer a bunch of different visual techniques that can help us a lot to achieve information insight. It has been widely used and applied with proven success in several areas of knowledge. We can say that thanks to visualization, any illiterate computer user is able to process huge volumes of data with the simple effort of an eye look.

Medicine is a vast scientific area whose main concern is health and welfare of human beings. Many computer applications focusing mainly image processing of X-rays, ultrasound or MRI (magnetic resonance imaging) have been produced. They help doctors to analyze these images, revealing details that sometimes our eyes cannot easily detect. Besides these applications, many others have been created, regarding other important aspects and issues of this scientific area, such as human body modeling, diagnosis decision support systems, data analysis or training systems (mostly in virtual reality environments). Computer graphics has been the essential key in all these applications or systems [11].

Though the computer graphics field is very important, another relevant point is the model that is used as a basis for the development of any graphic applications. The human body 
has been usually analyzed under a compartmented perspective. The models used in general to analyze and visualize its biological, morphological and/or biomedical data rarely represent their spatial interdependency in a suitable way. These models treat data in an almost totally focused and independent way. The human body systems and organs work as a complex machine, where each part depends strongly on the others. This dependence might be stronger or weaker to the system or organ depending on the overall patient condition. The doctor diagnoses an illness by comparing and analyzing information not only directly related to the mostly affected organ, but also to the body as a whole. In fact, the doctor performs a subtle spatial analysis, and therefore, executes a typical algebraic map operation in his mind, when diagnosing a patient. An illness might arouse different symptoms and physiological changes in systems/organs that are not directly related to the spatial location of it.

This paper presents a model to analyze the human body looking at the spatial interdependencies that exist between its several systems and organs. For the proposed model this paper also presents a case study in the hydrokinetic therapy and orthopedic area and points out the results that can be achieved with the implemented prototype.

This paper is divided into eight sections. The first one explains briefly what a cartographic model is while the second and third sections describe the general concepts that led us towards a cartographic approach in our model. The fourth section introduces its general architecture and the fifth the case studies used to evaluate the model and the adapted model. Finally, the sixth section presents the model evaluation and the seventh the main results we have achieved so far and the eighth the principal conclusion and future work.

\section{The cartographic model}

A cartographic model is defined as a simplified representation of the Earth's surface or any celestial body that can be expressed in an analytical form. For many years, cartography was only associated to the production of analogical maps. Nowadays, however, its domain grew and other fields of knowledge started to use it as a way to express information.

All the procedures associated to the cartographic modeling are based on data belonging to two or more maps. These maps are treated as geo-referenced information layers. Each layer must be spatially referenced. The way that they can be combined and analyzed is vast and varied. Normally, any combinations of these layers generate a new geo-referenced information layer [7].

Another important aspect is the analytical operation that is performed on a layer or layers [1]. The kind of algebraic operation of maps and the order that it is applied to layers creates different final results and as a consequence, different carto- graphic models. Analytical operations can be divided into three main categories: local, neighborhood and region. Local operations include reclassification and overlapping of layers using different logical operators (if, and, or, etc.). Neighborhood operations include filtering, buffering, proximity and connectivity analysis of areas in a map. Region operations determine areas and perimeters of areas of interest.

The cartographic model has been successfully exploited in many geographic information systems and is quite a standard model used to analyze and visualize data from the Earth Science knowledge field or with a strong spatial interdependency. Taking this into account, it is reasonable to say that a cartographic based model might also help to solve [3] the existing limitations usually present in human body data models [12].

\section{Applying cartography to the human body}

One main concept of a cartographic model is that its data can be arranged according to independent information layers. For instance, if we consider a country, its demography, average of literacy or age might be considered as information layers. These layers are normally geo-referenced, which means that they are referenced to some spot on the Earth's surface.

Because the human body is composed of several distinct systems and organs, and each one has a well-defined localization in its inside and a group of specific physiologies associated with, the conversion of its parts into information layers is almost straightforward. The systems and organs might be compared to states or counties, while physiological data, to census, rainfall or demographic data. The attributes are physiological parameters related to systems and organs, which can be measured and evaluated.

Each physiological parameter set is an information layer too. These attributes are "human-referenced", because they are spatially referenced to the centroid of a human body. Layers can be combined in different forms through algebraic maps operations.

The "human-referenced" physiological layers can contain data of varied type and nature-scalars, vectors, uniform, non-uniform, time-dependent, etc., depending on what organ or system it is related to. The nature and type of a specific layer changes accordingly to the human body system and organ to which it is related to.

Another facet is that any analytical combination performed between different "human-referenced" information layers can produce valuable results to help the diagnosis of human diseases. This means that using algebraic map operations on a human's body data might be useful to helps doctor's judgment. In fact, this is what he "implicitly" does when diagnosing any disease.

Finally, when "thinking" cartographically, we are intrinsically "seeing" a map. In terms of visualization, a map 


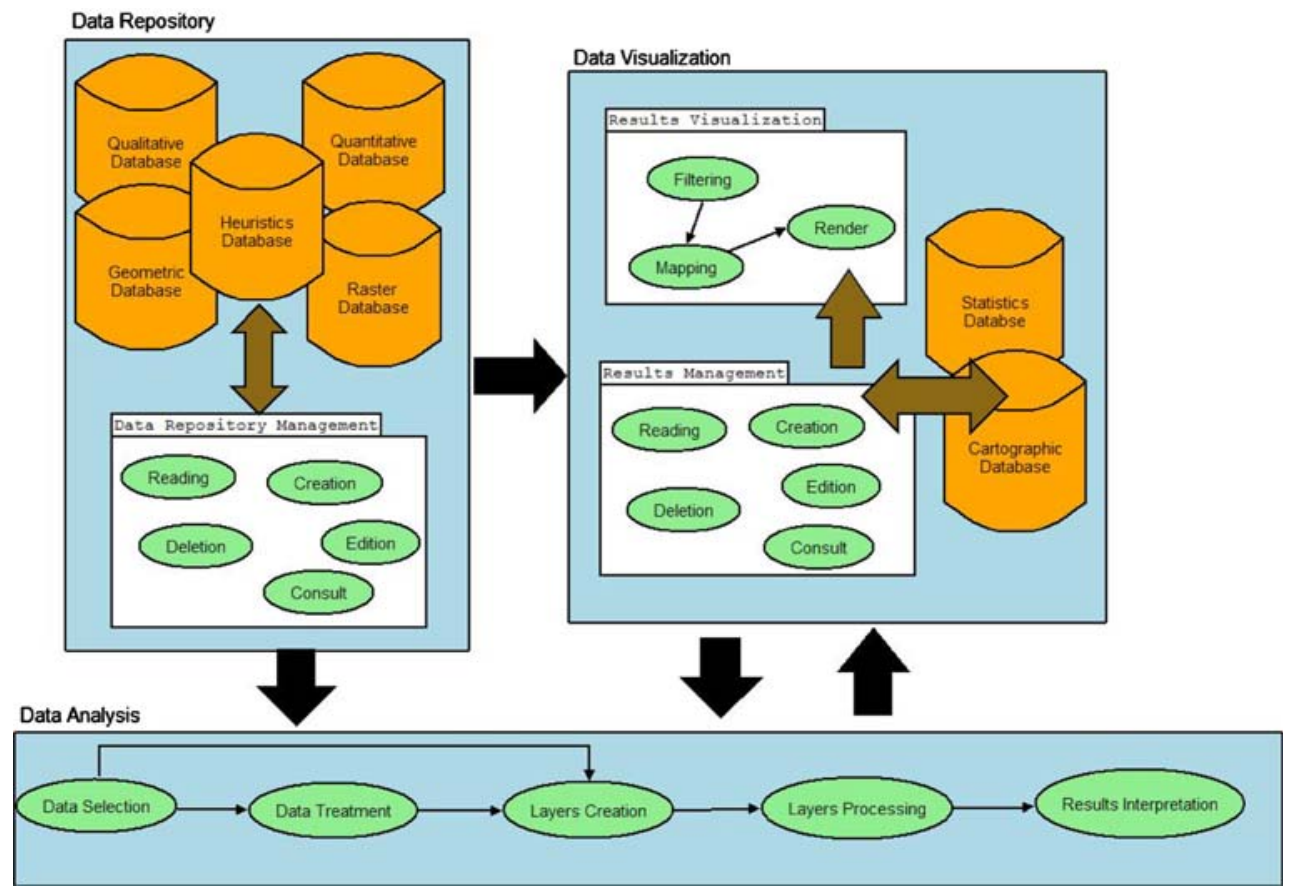

Fig. 1 CHUB's architecture

is usually a $2 \mathrm{D}$ or $3 \mathrm{D}$ surface representation, where counties and states are pseudo-colored, data elevation is represented with contour lines, relevant locations with symbols and resulting algebraic map operations with hachured textures. The visualization layers are set to "on" and "off" in order to focus a certain portion of geographical data. The map and the cartographic [14] approach is a very strong visual cue that should be also used to map non-geographic information. It helps to manage graphic complexity in visualizations and aids domain comprehension. The maps can be used and understood by a 4 year old children [13], with no need of previous training or experience. The use of cartographic metaphors can be of huge benefit in terms of information [4] understanding and human cognition and should be used by our model as far as possible [10].

\section{The CHUB's model architecture}

CHUB is a model $[3,4]$ that was developed taking into consideration the main principles of cartographic modeling. It structures data according to different layers of information. Each layer is related to a specific organ and/or system, and might contain geometric data and/or be associated indirectly to any quantitative, qualitative or image data, that are "humanreferenced". This "human-referenced" aspect means that all data are spatially referred to the centroid of the human body. This key concept is essential because of the cartographic nature of the model. CHUB has not been developed as a dynamic model. It is considered that dynamic issues related to human's body data, such as body movement, blood flow or heartbeat (besides others) will be accomplished by other models that should be used as a specialized extension to CHUB.

The model architecture includes three main components: (1) data repository; (2) data analysis and (3) data visualization. Figure 1 shows the general model design.

\section{Data repository}

This component is responsible for the management and storage of the qualitative (such as patient's address or name) and quantitative data (such as blood and urine exams results) related to the human body, the geometry 3D files describing different human organs and/or systems, the raster images from resulting exams (like, for instance, a X-ray or a tomography) and the heuristics that are used to evaluate and compare information layers by the model, applying different algebraic map operations on these layers. Both geometry 3D files and raster images are considered to be "human-referenced". In its management level, this component integrates stages such as creation, edition, consultation and deletion of records in the Qualitative and Heuristics Database, creation, reading and deletion of records in the Quantitative Database reading and deletion of raster files from the Raster Database, and reading of geometries from the Geometric Database.

An important feature is the possibility of visualizing "human-referenced" raster images of the human body. Nowadays, several exams generate 2D images of human body organs and/or systems. In order to CHUB be able to broadly support human body visualization and analysis, its data repo- 
sitory must also be able to manage information of this type. Each record in the Raster Database is composed of file location, its spatial reference, id of the type of exam that generated the raster file, data of creation and organ/system id.

Finally, all the results obtained in the Layers Processing stage from the Data Analysis component must have a group of rules that clearly define how these results must be evaluated and scored. In fact, these rules are a group of heuristics that are defined according to the goal of the human body analysis and visualization. The Heuristics Database contains rules that define how the results of algebraic map operations and/or mathematical/statistical models applied on the data should be combined, evaluated and scored.

\section{Data analysis}

This component is composed of the following stages: Data Selection, Data Treatment, Layers Creation, Layers Processing and Results Interpretation. It uses as input the data from the previous component, and sends its results to the Data Visualization component.

In the Data Selection stage, both the quantitative and the qualitative data that will be used to execute analysis and create information layers with attributes are identified, as well the $2 \mathrm{D}$ raster images that will be considered to compose the raster information layers. Besides this, the time range for data should also be indicated. This happens because both quantitative data and raster images might be related to different time moments (dates, for instance). In the case of quantitative data, a time moment might also be divided into several time intervals (hours, minutes or seconds, for instance). The quantitative data is sent to the Data Treatment stage, while the qualitative data and raster images to the Layers Creation stage.

In the Data Treatment stage, the selected quantitative data is initially evaluated in terms of noise and possible errors are eliminated. Data can be clustered according to different granularities. This granularity can be defined in terms of the number of the elements in the cluster (number of records in the quantitative data) or in terms of time range. In this stage, statistical or mathematical models to quantitative data is also identified and applied. These models evaluate data according to different scientific perspectives and goals.

Depending on what organ or system the resulting output data from the previous stage are related to, it might be assumed that data is spatially "human-referenced", because each organ or system has a well-defined spatial position within human body. Besides, the data can be related to different time moments and/or clusters. On the other hand, geometry data (stored in the Geometric Database) that describe different parts of the human body have a common spatial reference, to the scale of the human body. The same is valid to the 2D raster images (stored in the Raster Database). It is neces-

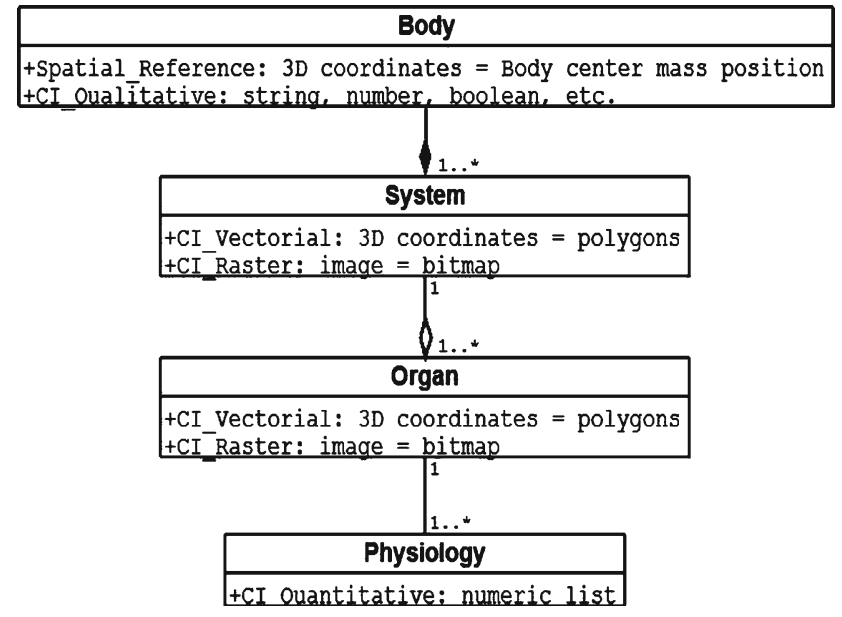

Fig. 2 Body's logic structure

sary that treated quantitative data from previous stage be also "human-referenced", so that it can be properly matched with other data. At this stage, this match is performed, and quantitative "human-referenced" information layers created. These layers are sent to the Data Visualization component and stored in the Statistics Database, being available for reuse by the system.

Besides, in this stage different "human-referenced" information layers are aggregated (and spatially interrelated between each other) in an information structure designated "Body" (see Fig. 2). This structure is internal to the model and is composed basically by pointers to different addresses inside Geometric, Qualitative, Raster and Statistics Databases. It also contains an explicit reference to the average centroid of the human body that serves as a spatial reference to the images, geometries and the treated quantitative data.

The stage Layers Processing is directly related to the application of algebraic maps operations to the "Body" information layers (created in the previous stage) and the evaluation of the obtained results. The user might be able to apply different spatial operations on treated data in order to extract more valuable information. The spatial combination of information layers can reveal and enhance important aspects of data correlation and interdependency. It is considered that the overlaying and reclassification spatial operations might be of special interest to the model.

The Results Interpretation stage allows the evaluation of all the options/decisions taken in the prior stages, integrating the results of the stage before. This evaluation generates results that might help doctors to diagnose or to monitor patient's health conditions.

\section{Data visualization}

This component is composed of two stages: Results Management and Results Visualization. The first stage is related to 
Table 1 Techniques, data and goals of visualization

\begin{tabular}{lll}
\hline Goal & Data & Technique \\
\hline Distribution & Scalars & Pseudo-colored 3D human body, 2D line chart, histogram \\
& Vectors & 3D hedgehogs, arrows \\
Difference, amplitude & Scalars & 2D bars chart 3D contour lines over the human body \\
& Vectors, tensors & Glyphs \\
\hline
\end{tabular}

\begin{tabular}{ll}
\hline Situation & Technique \\
\hline $\begin{array}{l}\text { Results of the overlaying operation } \\
\text { Results of the reclassification operation } \\
\text { Variation of } x, y \text { and } z \text { coordinates }\end{array}$ & Texture, pseudo-color, zoom, segmentation, transparency \\
\hline
\end{tabular}

Table 2 Visual cartographic oriented techniques tions. It is assumed that these techniques are applied to the $3 \mathrm{D}$ visual representation of the human body.

Finally, the stage Results Visualization is composed of three typical render pipeline stages: filtering, mapping techniques and render. The Filtering stage allows the end-user to choose portions of data to be visualized. The Mapping stage associates geometry data with visual attributes, according to the chosen visual technique. The Render stage turns geometry data into image.

\section{Cases studies}

A case study to validate and evaluate our model is required. Because of the model complexity, two different scenarios and case studies were taken into account: hydrokinetic therapy sessions and knee osteoarthritis diagnosis. Considering this approach, besides a more robust evaluation of our model, it will be possible to exploit better each of its potentialities. We are especially interested in evaluating our model capability to work as a diagnosis support tool (in this case, knee osteoarthritis) and an analytical tool (in this case, hydrokinetic therapy sessions). In both situations the cartographic oriented approach available in our model should proof its effectiveness.

In this section, it will be presented the applied/adapted model architecture and the consequential implemented prototype and its main achieved results.

\section{Scenarios}

\section{Knee osteoarthritis}

The knee is the intermediary articulation of the leg. It has possibly the most important role in the human motion. It is not easy to have the complete knowledge of everything that is related to this articulation and might affect it. It requires a combined effort between different disciplines such as the 

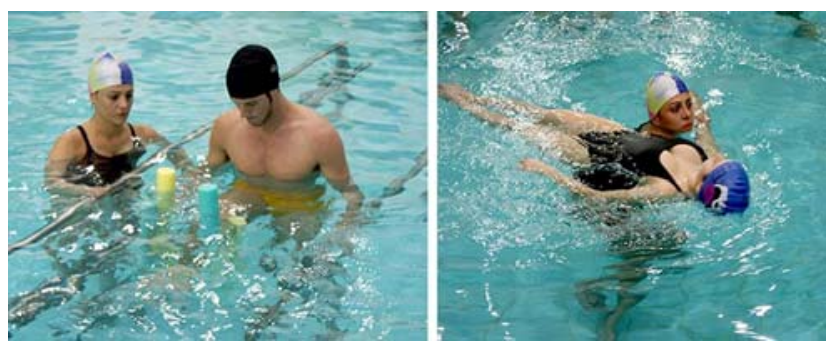

Fig. 3 Hydrokinetic therapy session [6]

anatomy, the biomechanics and the physiology surgery, with special emphasis on orthopedic surgery.

The muscle-skeletal evaluation [9] of the knee aims to detect problems and has three distinct phases: visual examination, touch examination and movement analysis. The first is basically the knee visual observation, so that any skin, bone or soft tissue anomaly is identified. The second is complementary to the first. The knee is palpated so that any temperature, flexibility or shape deformation is detected. The movement performed by the knee might be "active" or "passive" when its motion is being analyzed by the doctor. The third phase allows the evaluation of its stability and general health state.

Researchers believe that osteoarthritis is caused by a combination of factors, including overweight, the aging process, joint injury or stress, heredity, and muscle weakness. The pain in the knee triggered by any movement is a significant symptom. The patient slowly loses his knee motion, and it usually becomes swelled. The X-ray exams of the knee allow the evaluation of its inner space loss and any sclerosis or osteopathy formation. The osteoarthritis diagnosis is achieved after the muscle-skeletal evaluation of the knee and complementary exams such as X-rays, blood tests and MRIs.

\section{Hydrokinetic therapy sessions}

Hydrokinetic therapy sessions are composed of several and different techniques that are applied based on submersed human body movement. In fact, it is a kind of hydrophysiotherapy. Patients should perform several different therapeutic exercises and movements under water, according to their health needs and handicaps [6] (Fig. 3).

A special suit provided with electronic sensors is being developed to maximize session performance and allow an accurate monitoring and evaluation of patient's treatment response. It is clear the need of a well-tailored computer application that processes and records properly all data sent by sensors in real time. This computer application should also be able to offer effective and straightforward different data visualizations, which help therapists and doctors to have information insight, and easily evaluate patient's condition during a session and/or between sessions, working as a tool for diagnosis and decision support.

All captured data during a session will be sent to a computer in order to be properly processed. It is of great importance the creation of a suitable computer application that guarantees accurate and reliable data treatment. Besides, the huge amount of information demands powerful and comprehensive data visualization.

\section{Applied architecture}

Considering the two scenarios described in the previous section, our applied architecture should be adapted in order to process, treat and visualize properly hydrokinetic therapy sessions and knee osteoarthritis diagnosis. In the first case, it is considered to be also interesting to perform different statistical and historical evaluation on data, while in the knee osteoarthritis, the focus should be the usage of algebraic map operations to achieve the proper diagnosis.

The writing databases hold results of statistical evaluation of the hydrokinetic sessions (Statistic Database) and algebraic maps operations (Cartographic Database) performed on human-referenced information layers.

\section{Data repository component}

The Qualitative Database holds patients-personal information besides blood exams and X-rays graduations in terms of Alhbäck scale (it classifies the osteoarthritis gravity) while the Quantitative Database stores the hydrokinetic session's recording. All the geometry used by the prototype is kept inside the Geometric Database and is "human-referenced". The X-ray imagery of the patient is held by the Raster Database and is also "human-referenced". Inside the Heuristics Database are the logical descriptions of the decision tree that the doctor "mentally" and "implicitly" uses when diagnosing the knee osteoarthritis and the evaluation process that should be applied to analyze and estimate hydrokinetic therapy sessions (both in a single and a historic perspective). In fact, these descriptions are rules that identify how information layers should be matched and combined.

Patient's records are stored into a relational database in four tables. Patient's data include general information about the patient, such as his therapist, disease or age, and personalized physiological parameters such as his maximum permitted heartbeat value. These parameters are used for alarmist purposes. Hydrokinetic session recordings include patient's heartbeat, breathing frequency, hips, shoulders and spine rotations (in terms of pitch, yaw and roll) at each millisecond, besides suit identification number, patient's name and date and time of the recording. Each session might last $30 \mathrm{~min}$. Figure 4 illustrates the structure of a recording file. 


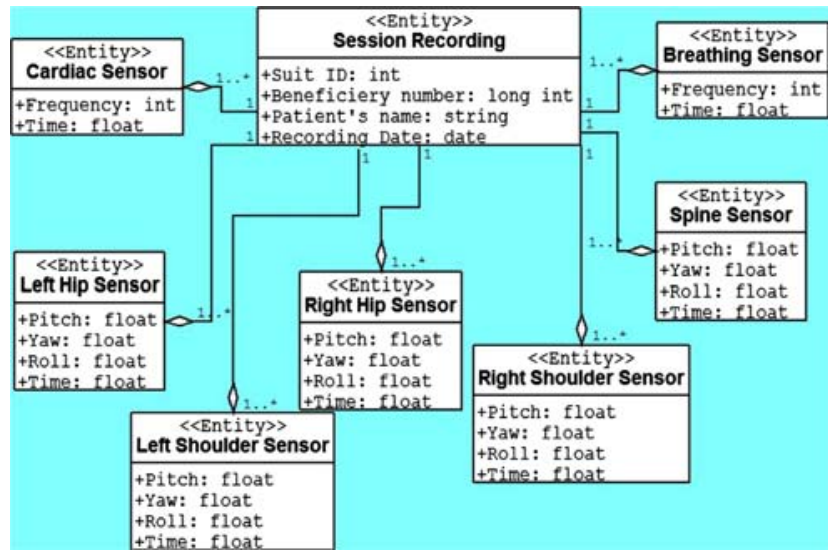

Fig. 4 Hydrokinetic session recording file

Eleven tables compose the Heuristics Database. The table Diagnosis is to define the rules to be used in the knee osteoarthritis diagnosis. The tables Causes, Symptoms and ClinicalExams hold, respectively, causes, symptoms and clinical exams that are directly related to the osteoarthritis disease diagnosis. Each of these tables associates an entry to some punctuation/score. By this means, the logical "mental" decision tree used by the doctor, with all its possible paths, is suitably represented. Besides these tables, in order to be feasible to establish the correspondence between the diseases, the diagnosis and its location within the human body (system, organ and physiology), additional tables are also present to hold this information. The table MapsAlgebra holds descriptions of algebraic map operations to be performed according the case. The other tables are used to evaluate the numerical analysis performed on hydrokinetic therapy recorded files. Figure 5 shows these tables scheme inside the Heuristics Database.

\section{Data analysis component}

In the Data Analysis component the statistical processing of data gathered along one and/or several hydrokinetic sessions is executed and the quantitative and qualitative patient data evaluated according the heuristics defined to diagnosis knee osteoarthritis. The layers are resultant of algebraic maps operations and are "human-referenced". Because a hydrokinetic session should also be analyzed and visualized in real time, data processing must attend the synchronism characteristics of a communication port reading. These aspects must be considered in both the Data Analysis and Results Visualization components.

If it is an analysis of a hydrokinetic therapy session, in the Data Selection stage, the patient and his related recording session files are chosen in order to be analyzed. Several sessions recordings and periods of time are available. If more than one session file is chosen, a historical evaluation of patient's data is performed; otherwise, a unique session is analyzed. In the Data Treatment stage, the noise is being deleted and data clustered according the chosen granularity in previous stage.

Otherwise, if it is a knee osteoarthritis diagnosis, in the Data Selection stage the patient should also be selected besides the causes, the symptoms and/or the clinical exams chosen. These supplementary data actually indicate the stage of the disease in the patient. In the Data Treatment stage, these data are combined and matched according to what is specified within the Heuristics Database. Figure 6 illustrates the classes that are involved in an osteoarthritis diagnosis processing.

In the Layer Creation stage data is arranged into layers. Figure 7 shows several layers of information and their interdependency when a hydrokinetic session is being evaluated. These layers are being processed in the Layer Processing stage. Maximum, minimum, medium, standard deviation and linear regression values both for clustered and global data are being calculated. The doctor or therapist might extract valuable information both from the treated data and the results obtained from this phase. He can also perform implicitly overlapping and reclassification spatial operations (algebraic maps operation) on data in order to achieve a knee osteoarthritis diagnosis evaluation.

Finally, in Results Interpretation stage, all the results from previous stages are consolidated and data sent to the Statistical Database if it was a hydrokinetic therapy session(s) analysis or to the Cartographic Database if it was a knee osteoarthritis diagnosis (the structure "Result" is therefore recorded for further reuse).

\section{Data visualization component}

Table 3 shows the correspondence between the situation/data in analysis and the proposed visualization technique. These visualizations are automatic generated.

Transparency is only used when it is a knee osteoarthritis diagnosis, so that the doctor may see inner parts of the articulation. Textures and colors are used to express the severity of the disease. Raster images of the radiographies are mapped with different hue, saturation and grey levels, so that some details might be better enhanced in the image.

\section{CHUB's model evaluation}

A prototype was implemented with VTK 5.0 [15] and KWWidgets [8] both open sources from Kitware Inc. The VTK is a three-dimensional (3D) computer graphics, image processing and visualization object oriented library. KWWidgets is an interface widget library. Both can be installed on 
Fig. 5 Heuristic database

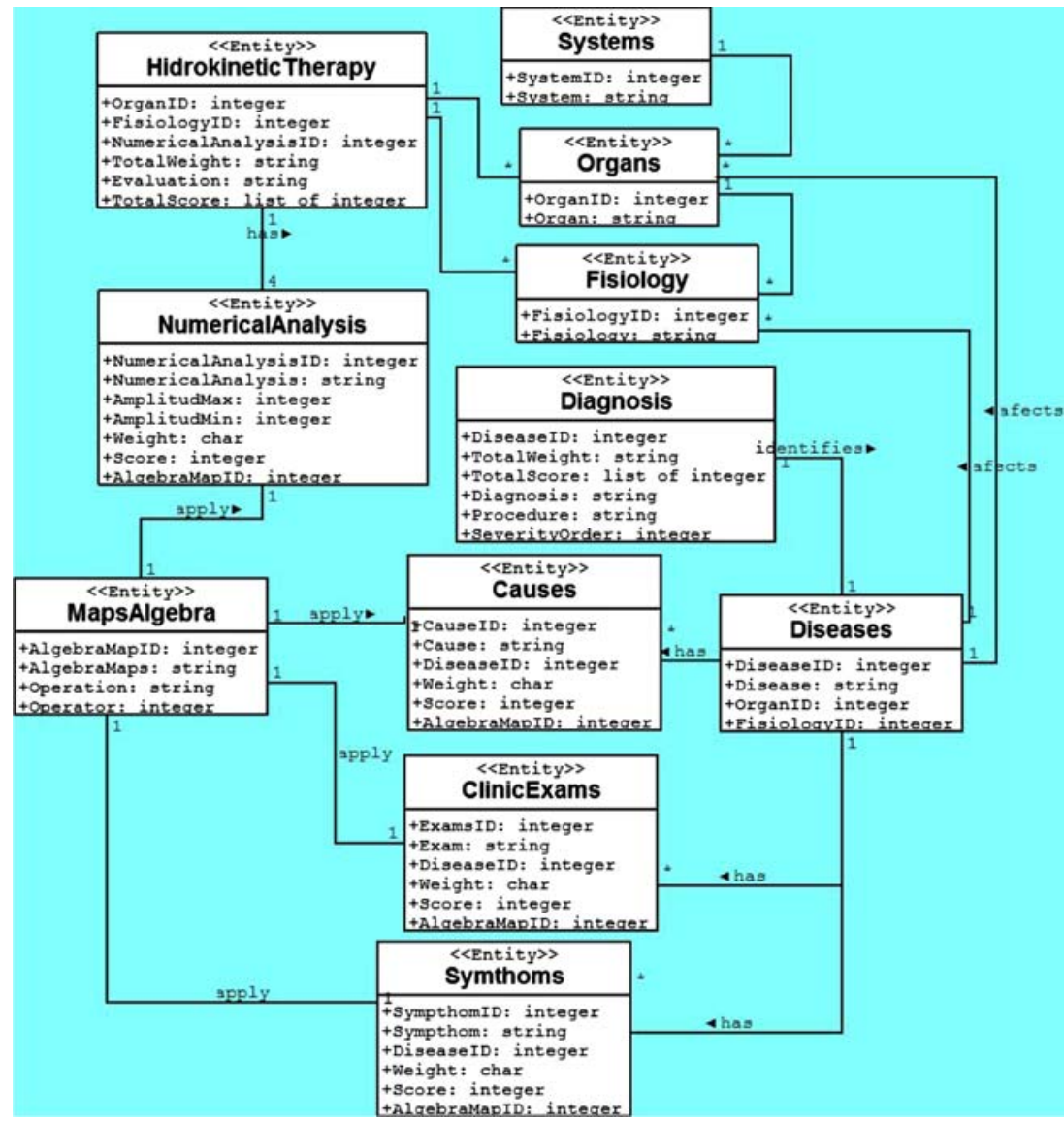

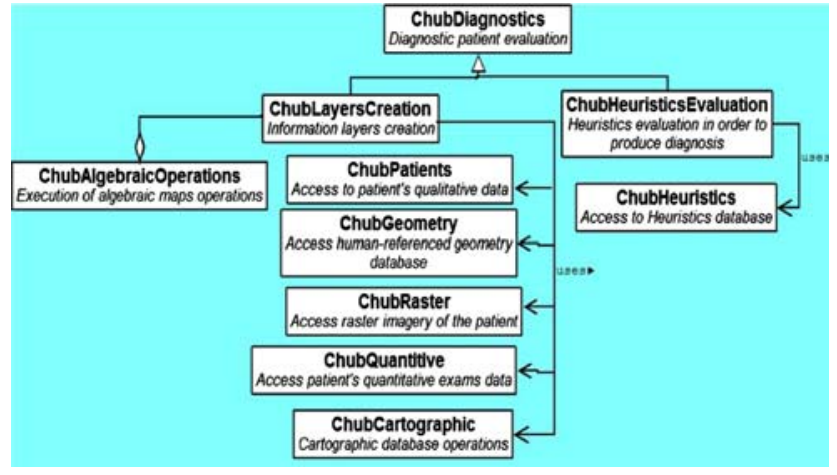

Fig. 6 CHUB's classes for osteoarthritis diagnosis

different platforms, such as Windows, Mac and various types of Unix/Linux. C++ was the programming language.

In order to evaluate our prototype two cases studies and approaches were considered. The first set of empirical tests were based on the Generic Evaluation Model (GEM) [2], proposed by Irène Buvat et al. The GEM includes the notion of hierarchical evaluation, identifies the components which have always to be defined when designing an evaluation protocol and shows the relationships that exist between these components. It was developed based on an exhaustive bibliographic study of the common procedures adopted to evaluate image processing and visualization in the medical field. This model considers that six different levels of evaluation should be attended:

- Level 1 is called evaluation of technical efficacy;

- Level 2 is called evaluation of the diagnostic accuracy efficacy of the method;

- Level 3 is called evaluation of the diagnostic thinking efficacy in evaluation of imaging technologies;

- Level 4 is the evaluation of the therapeutic efficacy of the imaging test;

- Level 5 is evaluation of the patient outcome efficacy. This corresponds to determining how the method affects patient outcome;

- Level 6 is the evaluation of the societal efficacy. A level 6 study would therefore aim at examining the benefit of the method for the society as a whole.

This approach was used to evaluate the CHUB in terms of knee osteoarthritis diagnosis (first case study). Adapting and applying GEM rules and levels to this situation, we identified six hypotheses that have to be tested and estimated in order to evaluate the model and consequently its prototype. These hypotheses focused only the osteoarthritis diagnosis because this is the portion of our CHUB prototype that actually takes advantage of our model cartographic approach. For each of these hypotheses we also defined the observer profile, the 


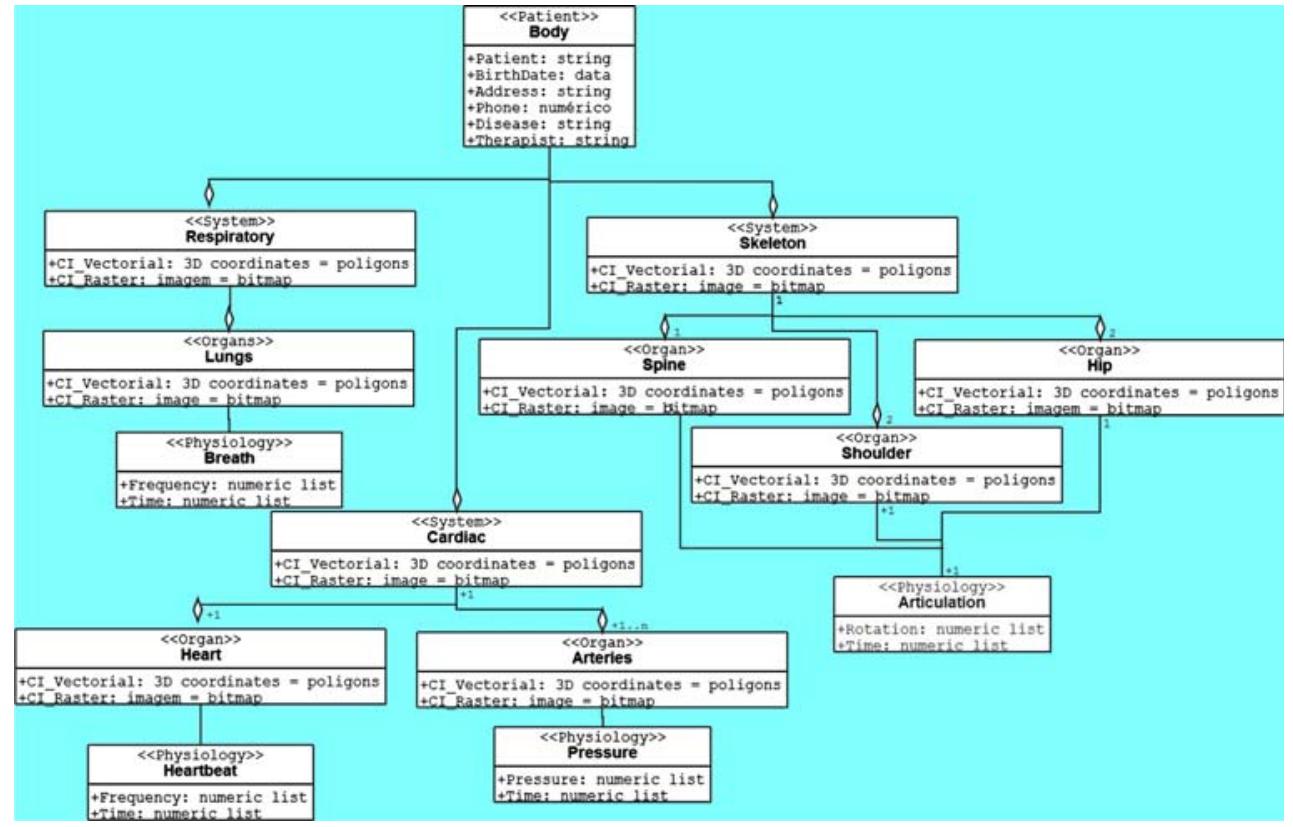

Fig. 7 Information layers in hydrokinetic therapy session(s) analysis

Table 3 Situation versus visualization techniques

\begin{tabular}{ll}
\hline Situation & Visualization technique \\
\hline Heartbeat & Line graph \\
Breathing frequency & Line graph \\
Rotations in articulations & 3D hedgehogs, 3D stereoscopic human body with animation and sensors tagged as symbols \\
Distribution of frequencies & Histogram \\
$\begin{array}{l}\text { Distribution of rotations } \\
\text { Knee osteoarthritis diagnosis }\end{array}$ & 3D human skeleton, hedgehogs \\
Overlapping operation & Texture, pseudo color, segmentation, transparency and zooming of a 3D stereoscopic human body \\
Knee osteoarthritis diagnosis & \\
Reclassification operation & Texture, pseudo color, segmentation, transparency and zooming of a 3D stereoscopic human body \\
Locations $(x, y, z)$ in the human body model & Contour lines, pseudo color, rotation and transparency of a 3D stereoscopic human skeleton \\
Alarms of danger situations & Sound, text and blinking effect \\
Radiographies & Pseudo-color and filtering \\
\hline
\end{tabular}

input data, the method and tasks to be applied and finally the quality index and a reference for results evaluation. The hypotheses were the following:

- Hypothesis 1. The appliance of cartographic and spatial operations on human-referenced information layers, allow knee osteoarthritis detection.

- Hypothesis 2. Besides what is referred to in hypothesis 1 , we can also properly achieve osteoarthritis diagnosis (severity grade).

- Hypothesis 3. Cartographic metaphors in results deliverance can help doctors to gain information insight.
- Hypothesis 4. CHUB can work properly as a diagnosis support tool.

- Hypothesis 5. CHUB usage actually eases the osteoarthritis knee diagnosis through its algebraic map operations and "human-referenced" data structures.

- Hypothesis 6. The CHUB can be successfully applied and used for the identification of other illnesses that can molest the knee.

A questionnaire divided into two parts was elaborated. It was completely based on GEM-oriented rules and levels and aimed our hypotheses assessment. In the first phase of the evaluation process, doctors had to fulfill the first part of the questionnaire while interviewing and diagnosing a patient. 


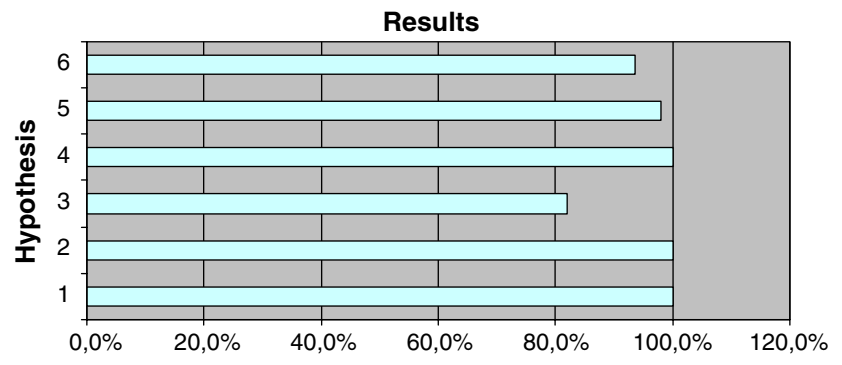

Fig. 8 Positive results for each hypothesis

In the second phase, doctors and therapists manipulated the prototype by exploring the different available features and functionalities and answered the missing part of the questionnaire. Thirty patients, presenting or not symptomatic knee osteoarthritis symptoms, were considered as test bay. Several patients and therapists were our potential prototype's users.

Further to these steps, questionnaire answers were numerically analyzed. Because the answers were directly correlated to one or more hypothesis acceptance or denial, it was possible to almost straightforwardly estimate their validation or not. Figure 8 shows the percentage of positive answers achieved by each hypothesis, while Table 4 shows the difference between these results and the proposed quality reference for each one of them. The differences with values equal to zero indicate that the hypothesis is accepted while a greater value means that it was fully affirmed (If we had negative values, this would mean a hypothesis full denial). For instance, hypothesis 5 was highly accepted, meaning that the usage of cartographic visual metaphors is really a good approach.

Based on empirical evidence resulted from this survey, we may conclude that human's body data actually have a very strong spatial interdependency. We could clearly validate this consideration while capturing the doctor's mental decision tree for the knee arthritis diagnosis or evaluating the sensors received data. For instance, according to the degree and strength of an articulation rotation, a significant variation occurs in patient's heartbeat. On the other hand, the standard procedure that any healthcare specialist follows while examining a patient is a combination of several algebraic map operations.

Our prototype evaluation pointed out to the full acceptance of our proposed hypotheses. We can state that the cartographic approach of our model introduces a satisfactory level of quality. The average difference of $11 \%$ indicates clearly that the usage of a cartographic oriented model can effectively help illness diagnosis and doctors achieve a better data insight.

The second approach focused the evaluation of CHUB as a general analytical and visualization tool for hydrokinetics therapy sessions. This evaluation was more informal
Table 4 Differences between hypotheses quality references and results

\begin{tabular}{llcc}
\hline Hypothesis & Reference $(\%)$ & Results $(\%)$ & Difference $(\%)$ \\
\hline 1 & 90.0 & 100.0 & 10 \\
2 & 90.0 & 100.0 & 10 \\
3 & 80.0 & 82.0 & 2 \\
4 & 90.0 & 100.0 & 10 \\
5 & 80.0 & 97.9 & 18 \\
6 & 80.0 & 93.7 & 14 \\
Average & 85.0 & 95.6 & 11 \\
\hline
\end{tabular}

and did not follow any special evaluation methodology. A questionnaire was elaborated focusing different aspects of the prototype, such as its functionalities, interaction characteristics and visualization outputs. This questionnaire was answered by 17 hydrokinetics physiotherapists after using CHUB's prototype for at least 15 days. The full acceptance of CHUB's solution was pointed out by $95 \%$ of the users. Some visualization techniques were not accepted such as the hedgehogs, and suggested the addition of some orientation glyph to the skeleton visualization.

\section{Principal results}

Our CHUB model and architecture has the advantage of supporting a fairly simple data processing and intuitive information visualization. The integration of different layers enables an effective data combination and therefore insight into data. Also, it is easy to establish the correspondence between body's quantitative data and the visualization results, because of the cartographic approach. Nevertheless, it has the disadvantages common to cartographic models-it is a static model. This can be overcome by the addition of an extension of a dynamic human's body model.

The prototype has some problems concerning fault tolerance and global delays (in real time sessions). During the first experiments in the context of the second case study (hydrokinetic therapy sessions) we notice a good acceptance and overall positive response. Some essential characteristics of the prototype such as statistic and historical data evaluation and session animated visualization (playing recorded sessions and seeing the patient's skeleton movement) were fully approved. The experiments in the context of the first case study (knee osteoarthritis diagnosis) have pointed out total approval of the considered hypothesis.

The application window is composed of four separators (database management, hydrokinetic therapy session or historic analysis and osteoarthritis diagnosis, respectively); each of them is divided into three distinct visualization areas. Figure 9 shows some of the visualizations available while Fig. 10 introduces the main visualization window to analyze 


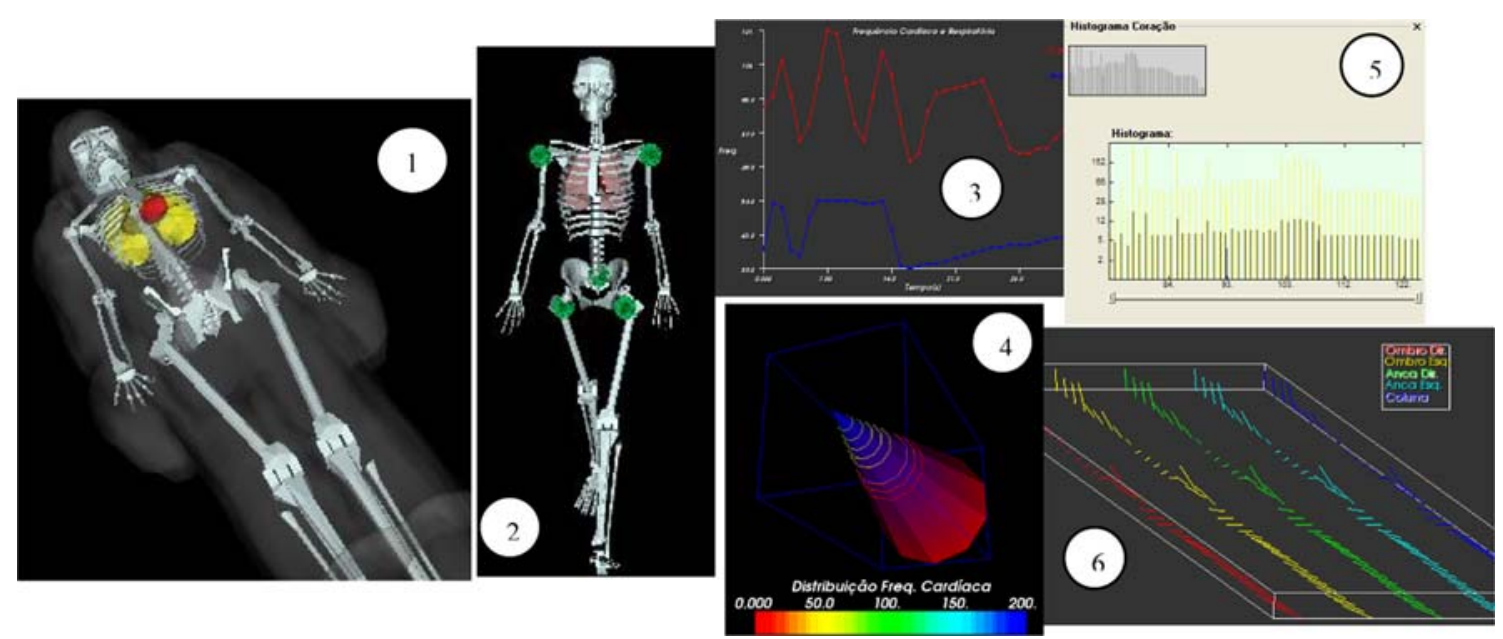

Fig. 9 Some snapshots of prototype's outputs: patient's skeleton with lungs and heart pseudo-colored according their health state (1) and in a "play" (2) of a session (sensors are mapped to the skeleton as green

glyphs), line graph of the heartbeat and breath frequencies (3), heartbeat distribution histogram (5) and mapped on a cone (4), hedgehog graph of the rotations of the articulations during a session (6)

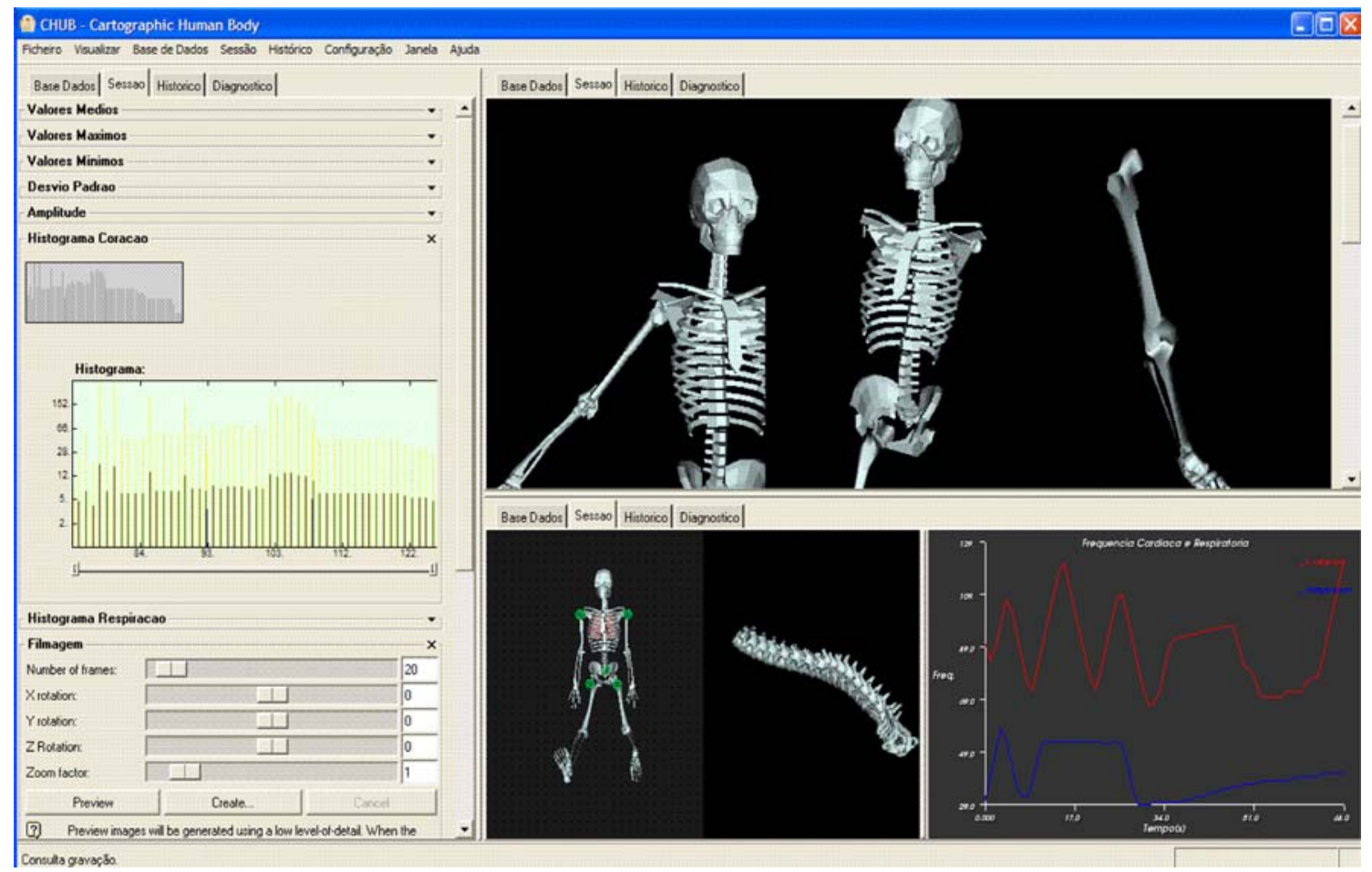

Fig. 10 Hydrokinetic session analysis and visualization

and view the treated data from hydrokinetics therapy recording sessions. The upper and lower left windows allows doctors/therapists to have different zooms and points of view of the rotations of patient's articulations, while the lower window works mainly as a heartbeat and movement monitor. Histograms, maximum, minimum, average, standard deviation and linear regression values, besides these views, work as a support tool for doctors/therapists to diagnose patient's overall condition.

Figure 11 shows the knee osteoarthritis diagnosis interface. According to the selected causes, symptoms and/or output exams, an ordered roll of plausible diagnosis is listed, besides a hierarchical tree of the suggested next therapeutic medical procedures. These views of the knee and its 


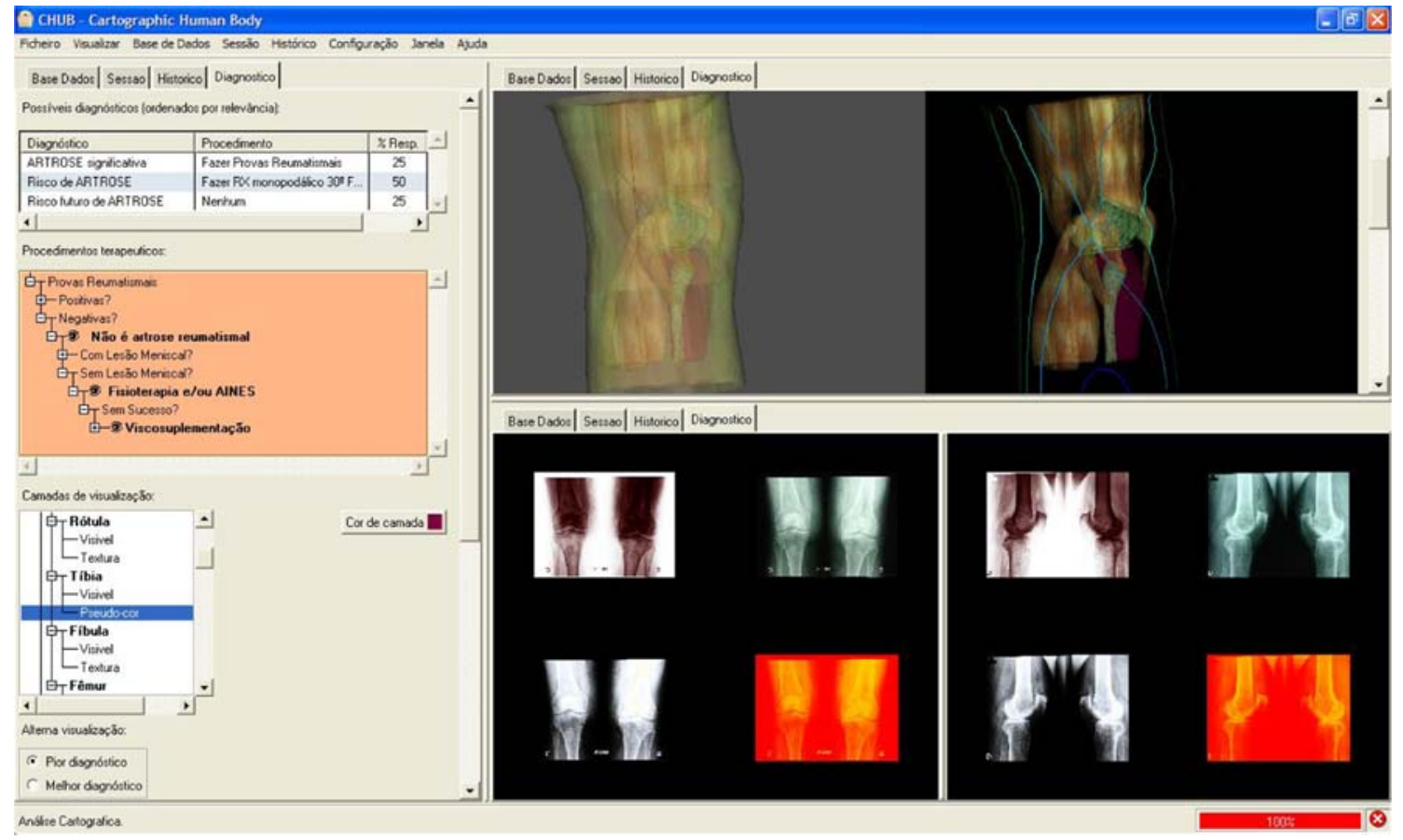

Fig. 11 Cartographic visualization and analysis

components are mapped with different textures, colors, degrees of transparency and contour lines.

\section{Conclusions and future work}

Nowadays, several applications are being developed taking into account our natural ability to think spatially. Cartographic models are a significant pillar of spatial analysis, and should not be underestimated.

Human's body data has a strong spatial interdependency and when a doctor diagnosis an illness, he "implicitly" perform some kind of algebraic map spatial operation on patient's physiological data. The human's body data should not be treated or processed in an independent and completely separated way. This is the most common mistake that medical oriented applications do. They focus and treat human's body data in an atomic way, not considering mostly, its strong spatial nature. This happens because they do not benefit from the usage of a cartographic-oriented model as a reference basis for their implementation.

CHUB is a model that takes into account all these considerations. It might be used as a reference model to any application that intends not only to analyze and visualize human's body data, but also with the proper adaptations, any organic data (which has the same spatial interdependency demands). It also takes advantage of using fairly simple and yet straightforward and expressive visual techniques that help doctors have information better insight.
Evaluation tests pointed out clearly that CHUB is a model that should be used as a reference to the implementation of any application whose main concern is human's body data analysis and visualization. Its validity leads us to conclude that a cartographic-oriented (both analytically and visually) approach introduces a new degree of effectiveness in human's body data. Furthermore, CHUB model should be used as a reference to any application that intends to analyze and visualize other organic beings, like mammals or birds, for instance.

Future work involves the addition of more heuristics to diagnosis other diseases, the improvement of the data structure used inside by the model and the implementation of it with GIS technology.

\section{References}

1. MacEchren A (2005) Exploring geovisualization. Elsevier, USA

2. Buvat I et al. (1999) The need to develop guidelines for the evaluation of medical processing procedures. Procedures SPIE Med Imaging 3661:1466-1477

3. Carvalho ES, Marcos AF, Santos MY, Brito PM (2007) Analysing and visualizing a human body cartographically. Proceedings of the $15^{\circ}$ Encontro Português de Computação Gráfica. Lisbon, 10-13 October, pp 1-8

4. Carvalho ES, Santos MY, Marcos AF (2007) CHUB — cartographic human body. Proceedings of IADIS-multi conference on computer science and information systems, Lisbon, 5-7 July, pp 27-34

5. Earnshaw R, Wiseman N (1992) Introductory guide to scientific visualization. Springer, Heidelberg 
6. Fiorelli A (2002) Hidrocinesioterapia: Princípios e Técnicas Terapêuticas. Colecções Plural. EDUSC, Santa Catarina

7. Korte G (2000) The GIS book, OnWord Press, USA

8. KWWidgets, http://www.kwwidgets.com/, visited on 25/03/08

9. Espregueira-Mendes J, Pessoa P, O Joelho (2006) Lidel Editions. Portugal

10. Peuquet D, Kraak M (2002) Geobrowsing: creative thinking and knowledge using geographic visualization. Informat Vis 1(1): 80 91

11. Russel M, Taylor II (2000) New visualization techniques.. ACM SIGGRAPH 34:1
12. Sato M (2001) Making a human body map. NatureInterface 4: $56-59$

13. Skupin A (2000) From metaphor to method: cartographic perspectives on information visualization. Proceedings IEEE InfoVis 2000, Utah, EUA, pp 91-97

14. Skupin A (2002) A cartographic approach to visualizing conference abstracts. IEEE CG\&A 22(1): 50-58

15. VTK, http://www.vtk.org/, visited on 25/03/08 